TRANSACTIONS OF THE

AMERICAN MATHEMATICAL SOCIETY

Volume 352, Number 8, Pages 3723-3742

S 0002-9947(00)02534- 4

Article electronically published on March 21, 2000

\title{
SEMICLASSICAL ANALYSIS OF GENERAL SECOND ORDER ELLIPTIC OPERATORS ON BOUNDED DOMAINS
}

\author{
E. N. DANCER AND J. LÓPEZ-GÓMEZ
}

\begin{abstract}
In this work we ascertain the semiclassical behavior of the fundamental energy and the ground state of an arbitrary second order elliptic operator, not necessarily selfadjoint, on a bounded domain. Our analysis provides us with substantial improvements of many previous results found in the context of quantum mechanics for $C^{\infty}$ perturbations of the Laplacian.
\end{abstract}

\section{INTRODUCTION}

In this paper we analyze the limiting behavior as $h \downarrow 0$ of the principal eigenpair to the following linear boundary value problem:

$$
h^{2} L \varphi+V(x) \varphi=\sigma \varphi \text { in } D, \quad \varphi=0 \quad \text { on } \partial D,
$$

where $D$ is a bounded domain of $\mathbf{R}^{N}$ with boundary $\partial D$ of class $C^{0,1}$ regularity, $L$ is a second order uniformly elliptic operator of the form

$$
L=-\sum_{i, j=1}^{N} a_{i j}(x) \partial_{i} \partial_{j}+\sum_{i=1}^{N} b_{i}(x) \partial_{i}+c(x),
$$

with

$$
a_{i j} \in C(\bar{D}), \quad b_{i}, c \in L^{\infty}(D), \quad i, j \in\{1, \ldots, N\},
$$

and $V \in L^{\infty}(D)$.

When $D=\mathbf{R}^{N}$, this problem has been analyzed in the context of quantum mechanics in the special cases where $L=-\Delta$ and $V \geq 0$ is a $C^{\infty}$ potential bounded away from zero at infinity and having a finite number of non-degenerate zeros (see [8]-[11], [22]-[25], and the references therein), and in the context of reaction-diffusion equations in some special cases where $L$ is selfadjoint or reducible to a selfadjoint operator, [18], [5. Some partial results were also given in Proposition 17.3 of [12].

In quantum physics $h \simeq 6.624 \times 10^{-27} \mathrm{erg} / \mathrm{sec}$ is Planck's constant. Except at atomic scales $h$ is very small, the length of the scales over which quantum effects are important being dependent on it. This fact provides us with a way of transition from classical to quantum mechanics, by comparing the classical limit when $h=0$ where all quantum effects have been neglected with the original quantum system through the semiclassical regime, where the Planck constant is assumed to be arbitrarily

Received by the editors August 13, 1997 and, in revised form, April 21, 1998.

2000 Mathematics Subject Classification. Primary 35P15, 35J10, 35B25.

(C)2000 American Mathematical Society 
small. When $V \geq 0$ is a $C^{\infty}$ potential bounded away from zero at infinity and having a finite number of non-degenerate zeros, then

$$
\sigma_{1}\left[-h^{2} \Delta+V\right]=E_{1} h+O\left(h^{2}\right) \quad \text { as } \quad h \downarrow 0,
$$

where $\sigma_{1}\left[-h^{2} \Delta+V\right]$ is the fundamental energy of $-h^{2} \Delta+V$ and $E_{1}$ is the fundamental energy of the associated harmonic oscillator obtained by localization at the wells of $V$. Moreover, the ground states concentrate into the set of zeros of $V$, and either there is a rapid eigenvalue degeneracy or the limiting ground states reside asymptotically in a single well (cf. Theorem 1.2 of [23]). It was the intention of Simon (see [22], p. 296) to study the case where $V$ vanishes on a manifold, but to the best of our knowledge, he only observed that if some of the zeros of $V$ are degenerate then $\sigma_{1}\left[-h^{2} \Delta+V\right]$ may go to zero faster than linearly, and that under enough hypotheses on the form of $V$ at the degenerate minima, it should be possible to get lower bounds for that decay. Some partial answers to these problems were given by Martínez and Rouleux in [19] and by one of the authors in [16]. In the context of Problem (1.1), it was shown in [16] that if the coefficients of the operator are Hölder continuous, $V \geq 0$, and $D_{0}:=V^{-1}(0)$ is a smooth domain, then

$$
\lim _{h \downarrow 0} \frac{\sigma_{1}^{D}\left[h^{2} L+V\right]}{h^{2}}=\sigma_{1}^{D_{0}}[L] .
$$

Hereafter, given a subdomain $D_{1} \subset D$ and an uniformly elliptic operator $\mathcal{L}$ in $D_{1}, \sigma_{1}^{D_{1}}[\mathcal{L}]$ stands for the principal eigenvalue of $\mathcal{L}$ in $D_{1}$ subject to homogeneous Dirichlet boundary conditions. In the special case when $L=-\Delta$, it was shown by one of the authors in [5] that in order to have (1.5) it suffices that $D_{0}$ supports a function of $W_{0}^{1,2}(D)$. In fact, if any such function with support in $D_{0}$ is trivial, then

$$
\lim _{h \downarrow 0} \frac{\sigma_{1}^{D}\left[h^{2} L+V\right]}{h^{2}}=\infty .
$$

In case (1.5) the fundamental energy decays quadratically, and in case (1.6) it decays at least linearly but slower than quadratically. In [19] it was shown that if $V \in C^{\infty}\left(\mathbf{R}^{N}\right)$ satisfies $V \geq 0, \liminf _{\|x\| \rightarrow \infty} V(x)>0, V^{-1}(0)=0$ and for $x \simeq 0$

$$
C^{-1}\|x\|^{\alpha} \leq V(x) \leq C\|x\|^{\alpha},
$$

where $C>0$ and $\alpha \geq 4$, then the decay rate of $\sigma_{1}\left[-h^{2} \Delta+V\right]$ as $h \downarrow 0$ is $h^{\frac{2 \alpha}{\alpha+2}}$. In fact, an asymptotic expansion was shown to exist for this special case.

The main goal of this paper is ascertaining the exact decay rate of the lowest eigenpair of (1.1) for some rather general classes of potentials in $L^{\infty}$. Our analysis will help to realize the nature and relationships between the several results obtained separately in the literature. We should point out that in our general setting we will have to deal with two serious difficulties. The first one comes from the lack of the symmetry of the differential operator, as we are working with general second order elliptic operators which in general do not possess a variational structure. The second one is inherent in the lack of regularity of the potential, under which we cannot hope to have the nice formal asymptotic expansions available in the literature for the case of smooth potentials by means of W.K.B. solutions. An extensive study of these asymptotic expansions can be found in the pioneering works by Helffer and Sjöstrand [9], [10], [11], which started a whole industry in this area. As general 
references for these and other related problems we refer to the books of Helffer 8 and Hislop and Sigal [14].

Our motivation to consider the general setting (1.1) comes originally from the analysis of singular perturbation results in the context of reaction-diffusion equations, [5], [16, [18, and from the analysis of the stability of the trivial state for a linear wave equation with damping, [17. When diffusivities and transport effects are uniformly small there naturally arises the problem of analyzing the decay rate of the principal eigenvalue and the behavior of the principal eigenfunctions, since they will eventually provide us with the behavior of the positive solutions for some general classes of non-linear singular perturbation problems.

The content of this paper is as follows. In Section 2 we recall some properties of principal eigenvalues and eigenfunctions that we are going to use throughout this work. We point out that some of them are new within our general setting. In fact, these properties are based upon a characterization of the strong maximum principle by means of the existence of positive strict supersolutions, which is a generalization of a result of [16] (cf. [1]), to which we refer for further details and references. In Section 3 we show that

$$
\lim _{h \downarrow 0} \sigma_{1}^{D}\left[h^{2} L+V\right]=\operatorname{ess} \inf _{D} V
$$

and that the limit of the ground states is concentrated in the region

$$
\left\{x \in D: V(x)>\operatorname{ess} \inf _{D} V\right\},
$$

i.e., we obtain the lowest order terms of the asymptotic expansion of the principal eigenpair of (1.1). These results are substantial improvements of Proposition 17.3 in the book of Hess [12] and of some other results found by the authors themselves (cf. 5] and [18) for selfadjoint operators. Even in the simplest situations when $V^{-1}\left(\operatorname{essinf}_{D} V\right)$ possesses several connected components, the ground states may reside asymptotically in only one of those components, instead of being asymptotically distributed in all of them (cf. Helffer [8, Helffer and Sjöstrand 9] and Simon 25]). Among other things, in Section 4 we show that the decay rates found by Martínez and Rouleux in [19] are still valid for our general setting. To be precise, let $a_{i j} \in W^{1, \infty}(D), b_{i}, c \in L^{\infty}(D), V \in L^{\infty}\left(\mathbf{R}^{N}\right)$,

$$
\left\{x \in D: V(x)=V_{L}\right\}=\left\{x_{0}, \ldots, x_{p}\right\}, \quad p \geq 0,
$$

and for each $i=0, \ldots, p$ let

$$
V(x)=V_{L}+\left\|x-x_{i}\right\|^{\alpha_{i}} g_{i}(\omega)+o\left(\left\|x-x_{i}\right\|^{\alpha_{i}}\right) \quad \text { as } x \rightarrow x_{i}, \quad \omega=\frac{x-x_{i}}{\left\|x-x_{i}\right\|},
$$

with $\alpha_{i}>0$ such that

$$
\alpha:=\alpha_{0}>\max _{1 \leq i \leq p} \alpha_{i}
$$

where each $g_{i}: S^{N-1} \rightarrow \mathbf{R}_{+}$is bounded and positive (bounded away from zero), $S^{N-1}$ stands for the $(N-1)$-dimensional unit sphere, and

$$
\liminf _{\|x\| \rightarrow \infty} V(x)>V_{L}
$$

Then we prove that

$$
\lim _{h \downarrow 0} \frac{\sigma_{1}^{D}\left[h^{2} L+V\right]-V_{L}}{h^{\frac{2 \alpha}{\alpha+2}}}=s\left(-\sum_{i, j=1}^{N} a_{i j}\left(x_{0}\right) \partial_{i} \partial_{j}+\|y\|^{\alpha} g_{0}(\omega)\right)>0,
$$


where, for a Schrödinger operator $\mathcal{L}$ in the whole of $\mathbf{R}^{N}, s(\mathcal{L})$ stands for the spectral bound of $\mathcal{L}$. Moreover, if $\Omega_{h}$ stands for the principal eigenfunction associated with $\sigma_{1}^{D}\left[h^{2} L+V\right]$ normalized so that $\left\|\Omega_{h}\right\|_{L^{2}(D)}=1$, then

$$
\lim _{h \downarrow 0}\left\|h^{\frac{N}{\alpha+2}} \Omega_{h}\left(x_{0}+h^{\frac{2}{\alpha+2}} \cdot\right)-\Psi_{0}(\cdot)\right\|_{L^{2}\left(\mathbf{R}^{N}\right)}=0,
$$

where $\Psi_{0}$ is the principal eigenfunction associated with

$$
s\left(-\sum_{i, j=1}^{N} a_{i j}\left(x_{0}\right) \partial_{i} \partial_{j}+\|y\|^{\alpha} g_{0}(\omega)\right)
$$

normalized so that $\int_{\mathbf{R}^{N}} \Psi_{0}^{2}(y) d y=1$. We should point out that the corresponding results of Martínez and Rouleux were shown to hold for the very special case when $L=-\Delta, V \in C^{\infty}, p=0$ and $\alpha \geq 4$. Notice that the decay rate $\frac{2 \alpha}{\alpha+2}$ approaches 2 as $\alpha \rightarrow \infty$. Hence, it is rather natural to predict that whenever the potential vanishes on an open subset of $D$ the decay rate to $V_{L}$ of the fundamental energy will be $h^{2}$. This analysis will be accomplished in Section 5 , where it will be shown that

$$
\lim _{h \downarrow 0} \frac{\sigma_{1}^{D}\left[h^{2} L+V\right]-V_{L}}{h^{2}}=\sigma_{1}^{D_{0}}[L]
$$

where

$$
D_{0}=\left\{x \in D: V(x)=V_{L}\right\},
$$

and that the limiting ground state is concentrated within $D_{0}$. To the best of our knowledge, this last result is completely unknown in the quantum mechanics literature.

\section{THE MAXIMUM PRINCIPLE.}

MAIN PROPERTIES OF THE PRINCIPAL EIGENVALUES

In this section we give an extension of Theorem 2.5 in [16] to cover our setting here, and then we infer some basic properties of principal eigenvalues which will be used throughout this paper. We will use the natural product order on $L^{p}(D) \times L^{p}(\partial D)$. Recall that $p>N$ implies $W^{2, p}(D) \subset C^{2-\frac{N}{p}}(\bar{D})$ with compact embedding, and that each $u \in W^{2, p}(D)$ is a.e. twice classically differentiable in $D$ (e.g., Theorem VIII.1 of [26]).

Suppose that $p>N$. Then $u \in W^{2, p}(D)$ is said to be strongly positive if $u(x)>0$ for $x \in D$ and $\partial_{n} u(x)<0$ for all $x \in \partial D$ with $u(x)=0$, where $n$ is the outward unit normal on $\partial D$. The operator $L$ is said to satisfy the strong maximum principle in $D$ if $p>N, u \in W^{2, p}(D)$, and $(L u, u)>(0,0)$ in $L^{p}(D) \times L^{p}(\partial D)$ imply that $u$ is strongly positive. Consider the eigenvalue problem

$$
L u=\sigma u \text { in } D, \quad u=0 \text { on } \partial D,
$$

in $W^{2, p}(D)$ and let $L_{p}$ denote the closure of the operator $\left.L\right|_{W_{0}^{2, p}(D)}$ in $L^{p}(D)$. Then (2.1) can be reformulated as the eigenvalue equation

$$
L_{p} u=\sigma u \quad \text { in } L^{p}(D) .
$$

It is an easy consequence of standard elliptic regularity theory that the spectrum and the eigenspaces of $L_{p}$ are independent of $p>N$. Moreover, from the strong maximum principle and the generalization of the Krein-Rutman Theorem of [21] together with Theorem 3 in [20], the following result holds (cf. Section 2 of [1]). 
Theorem 2.1. There exists a least eigenvalue of (2.2), denoted by $\sigma_{1}^{D}[L]$ and called the principal eigenvalue of $L$ in $D$. This eigenvalue is simple and possesses a unique eigenfunction, up to multiplicative constants, which can be taken positive, the socalled principal eigenfunction of $L$ in $D$. Moreover, the principal eigenfunction is strongly positive, and $\sigma_{1}^{D}[L]$ is the only eigenvalue of 2.2 possessing a positive eigenfunction. Furthermore, any other eigenvalue $\sigma$ of (2.2) satisfies

$$
\operatorname{Re} \sigma>\sigma_{1}^{D}[L]
$$

and $\left(L_{p}+\lambda\right)^{-1} \in \mathcal{L}\left(L^{p}(D)\right)$ is positive, compact and irreducible for $\lambda>\sigma_{1}^{D}[L]$.

If $p>N$, a function $\bar{u} \in W^{2, p}(D)$ is said to be a positive strict supersolution of $L$ in $D$ if $\bar{u} \geq 0$ and $(L \bar{u}, \bar{u})>(0,0)$ in $L^{p}(D) \times L^{p}(\partial D)$. From the strong maximum principle it is easily seen that any positive strict supersolution is strongly positive. Moreover, the following characterization of the strong maximum principle holds (cf. Theorem 2.5 in 16] and Theorem 2.4 in [1]).

Theorem 2.2. The following assertions are equivalent:

(i) $\sigma_{1}^{D}[L]>0$;

(ii) L possesses a positive strict supersolution in D;

(iii) L satisfies the strong maximum principle in $D$.

From this characterization we can readily get the following properties of $\sigma_{1}^{D}[L]$ which will be used throughout this work. For selfadjoint operators, these properties are easily obtained from the variational characterization of the principal eigenvalue.

Theorem 2.3. (i) Monotonicity with respect to the potential: Let $V_{1}, V_{2} \in L^{\infty}(D)$ be such that $V_{1} \leq V_{2}$ and $V_{1}<V_{2}$ on a set of positive measure. Then

$$
\sigma_{1}^{D}\left[L+V_{1}\right]<\sigma_{1}^{D}\left[L+V_{2}\right] .
$$

(ii) If $D_{1}$ is a proper subdomain of $D$ with $\partial D_{1}$ of class $C^{0,1}$, then

$$
\sigma_{1}^{D_{1}}[L]>\sigma_{1}^{D}[L]
$$

Proof. (i) Let $\varphi_{1}$ be the principal eigenfunction associated with $\sigma_{1}^{D}\left[L+V_{1}\right]$. Then

$$
\left(L+V_{2}\right) \varphi_{1}=\sigma_{1}^{D}\left[L+V_{1}\right] \varphi_{1}+\left(V_{2}-V_{1}\right) \varphi_{1}>\sigma_{1}^{D}\left[L+V_{1}\right] \varphi_{1}
$$

on a set of positive measure, and hence $\varphi_{1}$ is a positive strict supersolution of $L+V_{1}-\sigma_{1}^{D}\left[L+V_{1}\right]$. Thus, thanks to Theorem 2.2] we find that

$$
\sigma_{1}^{D}\left[L+V_{1}-\sigma_{1}^{D}\left[L+V_{1}\right]\right]>0 .
$$

This relation implies (2.3).

(ii) Let $\varphi$ denote the principal eigenfunction associated with $\sigma_{1}^{D}[L]$. Then

$$
\left(L-\sigma_{1}^{D}[L]\right) \varphi=0
$$

in $D_{1}$ and $\varphi>0$ on $\partial D_{1}$. Thus, $\varphi$ is a positive strict supersolution of $L-\sigma_{1}^{D}[L]$ in $D_{1}$, and hence it follows from Theorem 2.2 that

$$
\sigma_{1}^{D_{1}}\left[L-\sigma_{1}^{D}[L]\right]>0 .
$$

This relation implies (2.4). 


\section{LiMITING BEHAVIOR OF THE PRINCIPAL EIGENPAIR}

The following result shows the behavior of the principal eigenvalue.

Theorem 3.1. Assume (1.3) and $V \in L^{\infty}(D)$. Then,

$$
\lim _{h \downarrow 0} \sigma_{1}^{D}\left[h^{2} L+V\right]=V_{L},
$$

where

$$
V_{L}:=\operatorname{ess} \inf _{D} V .
$$

Proof. By the monotonicity of the principal eigenvalue with respect to the potential we have

$$
\sigma_{1}^{D}\left[h^{2} L+V\right] \geq h^{2} \sigma_{1}^{D}[L]+V_{L}
$$

and hence

$$
\liminf _{h \downarrow 0} \sigma_{1}^{D}\left[h^{2} L+V\right] \geq V_{L} .
$$

Since $V \in L^{\infty}(D), V \in L^{1}(D)$. Hence, by the Lebesgue differentiation theorem, for almost every point $x_{0} \in D$ the following holds:

$$
\lim _{h \downarrow 0} \frac{1}{\mu\left(B_{h}\left(x_{0}\right)\right)} \int_{B_{h}\left(x_{0}\right)}\left|V(x)-V\left(x_{0}\right)\right| d x=0,
$$

where $B_{h}\left(x_{0}\right)$ denote the ball of radius $h$ centered at $x_{0}$ and $\mu(\cdot)$ is the Lebesgue measure in $\mathbf{R}^{N}$. Thus, by a change of variable, for each $R>0$ we have

$$
\lim _{h \downarrow 0} \int_{B_{R}}\left|V\left(x_{0}+h x\right)-V\left(x_{0}\right)\right| d x=0 \quad \text { for almost all } x_{0} \in \mathbf{R}^{N},
$$

where $B_{R}:=B_{R}(0)$. Therefore, for each $p \in[1, \infty)$

$$
\lim _{h \downarrow 0} \int_{B_{R}}\left|V\left(x_{0}+h x\right)-V\left(x_{0}\right)\right|^{p} d x=0 \quad \text { for almost all } x_{0} \in \mathbf{R}^{N},
$$

since $V \in L^{\infty}(D)$. Now, fix $\delta>0$ arbitrary and $p>N$. By (3.3) there exists $x_{0} \in D$ such that

$$
\lim _{h \downarrow 0} \int_{B_{R}}\left|V\left(x_{0}+h x\right)-V\left(x_{0}\right)\right|^{p} d x=0 \quad \text { and } \quad V\left(x_{0}\right) \leq V_{L}+\delta .
$$

By rescaling the spatial variable, we find that

$$
\sigma_{1}^{B_{R}}\left[-\sum_{i, j=1}^{N} a_{i j}\left(x_{0}\right) \partial_{i} \partial_{j}+V\left(x_{0}\right)\right]=\sigma_{1}^{B_{1}}\left[-\frac{1}{R^{2}} \sum_{i, j=1}^{N} a_{i j}\left(x_{0}\right) \partial_{i} \partial_{j}+V\left(x_{0}\right)\right] .
$$

Moreover,

$$
\begin{aligned}
\sigma_{1}^{B_{1}}[ & \left.-\frac{1}{R^{2}} \sum_{i, j=1}^{N} a_{i j}\left(x_{0}\right) \partial_{i} \partial_{j}+V\left(x_{0}\right)\right] \\
& =\frac{1}{R^{2}} \sigma_{1}^{B_{1}}\left[-\sum_{i, j=1}^{N} a_{i j}\left(x_{0}\right) \partial_{i} \partial_{j}\right]+V\left(x_{0}\right) \rightarrow V\left(x_{0}\right)
\end{aligned}
$$


as $R \uparrow \infty$, and hence

$$
\lim _{R \uparrow \infty} \sigma_{1}^{B_{R}}\left[-\sum_{i, j=1}^{N} a_{i j}\left(x_{0}\right) \partial_{i} \partial_{j}+V\left(x_{0}\right)\right]=V\left(x_{0}\right) .
$$

Fix $R=R(\delta)$ such that

$$
\sigma_{1}^{B_{R}}\left[-\sum_{i, j=1}^{N} a_{i j}\left(x_{0}\right) \partial_{i} \partial_{j}+V\left(x_{0}\right)\right] \leq V\left(x_{0}\right)+\frac{\delta}{2} .
$$

Now, for $h>0$ sufficiently small let $\mathcal{L}_{h}$ denote the differential operator

$$
\mathcal{L}_{h}:=-\sum_{i, j=1}^{N} a_{i j}\left(x_{0}+h x\right) \partial_{i} \partial_{j}+h \sum_{i=1}^{N} b_{i}\left(x_{0}+h x\right) \partial_{i}+h^{2} c\left(x_{0}+h x\right)+V\left(x_{0}+h x\right)
$$

with domain

$$
\mathcal{D}\left(\mathcal{L}_{h}\right):=W_{0}^{1, p}\left(B_{R}\right) \cap W^{2, p}\left(B_{R}\right) .
$$

We claim that these operators converge as $h \downarrow 0$ to the operator

$$
\mathcal{L}_{0}:=-\sum_{i, j=1}^{N} a_{i j}\left(x_{0}\right) \partial_{i} \partial_{j}+V\left(x_{0}\right), \quad \mathcal{D}\left(\mathcal{L}_{0}\right):=W_{0}^{1, p}\left(B_{R}\right) \cap W^{2, p}\left(B_{R}\right),
$$

in the generalized sense of Kato ([15], IV.2.4). Indeed, for each $u \in W^{2, p}\left(B_{R}\right)$ we have

$$
\begin{aligned}
\left\|\mathcal{L}_{h} u-\mathcal{L}_{0} u\right\|_{L^{p}\left(B_{R}\right)} \leq & \left(\max _{1 \leq i, j \leq N}\left\|a_{i j}\left(x_{0}+h \cdot\right)-a_{i j}\left(x_{0}\right)\right\|_{L^{\infty}\left(B_{R}\right)}\right. \\
& \left.+h \max _{1 \leq i \leq N}\left\|b_{i}\right\|_{L^{\infty}(D)}+h^{2}\|c\|_{L^{\infty}(D)}\right)\|u\|_{W^{2, p}\left(B_{R}\right)} \\
& +\|u\|_{L^{\infty}\left(B_{R}\right)}\left\|V\left(x_{0}+h \cdot\right)-V\left(x_{0}\right)\right\|_{L^{p}\left(B_{R}\right)},
\end{aligned}
$$

where we have used the embedding $W^{2, p}\left(B_{R}\right) \subset C\left(\bar{B}_{R}\right)$, which is compact. Moreover, since $a_{i j} \in C(\bar{D})$, for $h$ sufficiently small $a_{i j}\left(x_{0}+h x\right)$ is uniformly close to $a_{i j}\left(x_{0}\right)$ in $B_{R}$. Therefore, it follows from (3.4) and (3.6) that for any $\epsilon>0$ there exists $h_{0}>0$ such that

$$
\left\|\mathcal{L}_{h} u-\mathcal{L}_{0} u\right\|_{L^{p}\left(B_{R}\right)} \leq \epsilon\|u\|_{W^{2, p}\left(B_{R}\right)}
$$

for all $h \in\left(0, h_{0}\right)$ and $u \in W^{2, p}\left(B_{R}\right)$. This shows the convergence of the graphs of the $\mathcal{L}_{h}$ to the graph of $\mathcal{L}_{0}$, and hence the previous claim is shown. Therefore, due to Kato 15, IV.3.5, any finite system of eigenvalues of $\mathcal{L}_{h}$ changes with $h$ continuously towards the corresponding eigenvalues of $\mathcal{L}_{0}$ just as in the finite-dimensional case. In particular, due to (3.5), for $h$ small we have

$$
\sigma_{1}^{B_{R}}\left[\mathcal{L}_{h}\right] \leq V\left(x_{0}\right)+\delta .
$$

Rescaling the spatial variables, this relation gives

$$
\sigma_{1}^{B_{R_{h}}\left(x_{0}\right)}\left[h^{2} L+V(x)\right] \leq V\left(x_{0}\right)+\delta \leq V_{L}+2 \delta,
$$

and therefore, the monotonicity of the principal eigenvalue with respect to the domain gives

$$
\sigma_{1}^{D}\left[h^{2} L+V(x)\right] \leq \sigma_{1}^{B_{R_{h}}\left(x_{0}\right)}\left[h^{2} L+V(x)\right] \leq V_{L}+2 \delta .
$$


As this can be done for any $\delta>0$, we find that

$$
\limsup _{h \downarrow 0} \sigma_{1}^{D}\left[h^{2} L+V(x)\right] \leq V_{L} .
$$

This completes the proof.

Theorem 3.1 provides us with the asymptotic behavior of the mapping $\lambda \rightarrow$ $\sigma_{1}^{D}[L+\lambda V]$ at $\pm \infty$. Indeed,

$$
\lim _{\lambda \uparrow \infty} \frac{\sigma_{1}^{D}[L+\lambda V]}{\lambda}=\operatorname{ess} \inf _{D} V, \quad \lim _{\lambda \downarrow-\infty} \frac{\sigma_{1}^{D}[L+\lambda V]}{\lambda}=-\operatorname{ess} \sup _{D} V .
$$

Thus, if in addition we assume

$$
\text { ess } \inf _{D} V<0, \quad \operatorname{ess} \sup _{D} V>0, \quad \sigma_{1}^{D}[L]>0,
$$

then due to the concavity of $\lambda \rightarrow \sigma_{1}^{D}[L+\lambda V]$ we find that the weighted boundary value problem

$$
L \varphi=\lambda V \varphi \text { in } D, \quad \varphi=0 \text { on } \partial D,
$$

possesses two principal eigenvalues; one negative and the other positive. This result slightly improves the main theorem of Hess and Kato [13, obtained for $V \in C(\bar{D})$.

The following result shows that the principal eigenfunction associated with $\sigma_{1}^{D}\left[h^{2} L+V\right]$ converge in $L^{2}(D)$ to 0 in the region $\left\{x \in D: V(x)>V_{L}\right\}$ as $h \downarrow 0$.

Theorem 3.2. Suppose $a_{i j} \in W^{1, \infty}(D)=C^{0,1}(\bar{D})$ and $b_{i}, c, V \in L^{\infty}(D)$. Let $\Omega_{h}>0$ denote the principal eigenfunction associated with $\sigma_{1}^{D}\left[h^{2} L+V\right]$, normalized so that

$$
\int_{D}\left|\nabla \Omega_{h}\right|^{2}=1
$$

Then, for any sequence $h_{n} \downarrow 0$, as $n \uparrow \infty$, there exists a subsequence (relabeled by n) such that

$$
\lim _{n \rightarrow \infty} \Omega_{h_{n}}=\Omega_{0} \quad \text { in } L^{2}(D),
$$

for some $\Omega_{0} \in L^{2}(D)$ such that

$$
\Omega_{0}=0 \quad \text { in } \quad D_{+}:=\left\{x \in D: V(x)>V_{L}\right\} .
$$

In particular,

$$
\lim _{h \downarrow 0}\left\|\Omega_{h}\right\|_{L^{2}\left(D_{+}\right)}=0 .
$$

Proof. By Theorem 3.1 we already know that

$$
\lim _{h \downarrow 0} \sigma_{1}^{D}\left[h^{2} L+V\right]=V_{L} .
$$

If we set

$$
B_{j}:=\sum_{i=1}^{N} \partial_{i}\left(a_{i j}\right)+b_{j}, \quad j=1, \ldots, N
$$

the equation

$$
h^{2} L \Omega_{h}+V \Omega_{h}=\sigma_{1}^{D}\left[h^{2} L+V\right] \Omega_{h}
$$


becomes

$$
-h^{2} \sum_{i, j=1}^{N} \partial_{i}\left(a_{i j} \partial_{j} \Omega_{h}\right)+h^{2} \sum_{j=1}^{N} B_{j} \partial_{j} \Omega_{h}+h^{2} c \Omega_{h}+V \Omega_{h}=\sigma_{1}^{D}\left[h^{2} L+V\right] \Omega_{h} .
$$

Multiplying (3.11) by $\Omega_{h}$ and integrating on $D$ yields

$$
\begin{gathered}
h^{2} \sum_{i, j=1}^{N} \int_{D} a_{i j} \partial_{i} \Omega_{h} \partial_{j} \Omega_{h}+h^{2} \sum_{j=1}^{N} \int_{D} B_{j} \Omega_{h} \partial_{j} \Omega_{h}+\int_{D}\left(h^{2} c+V\right) \Omega_{h}^{2} \\
=\sigma_{1}^{D}\left[h^{2} L+V\right] \int_{D} \Omega_{h}^{2} .
\end{gathered}
$$

By (3.6), $\left(\Omega_{h}\right)$ is bounded in $W_{0}^{1,2}(D)$. Thus, from any sequence $\left(h_{n}\right), n \geq 1$, $h_{n} \downarrow 0$, we can extract a subsequence, relabeled by $n$, such that

$$
\lim _{n \rightarrow \infty}\left\|\Omega_{h_{n}}-\Omega_{0}\right\|_{L^{2}(D)}=0
$$

for some $\Omega_{0} \in L^{2}(D)$. Since $B_{j} \in L^{\infty}(D)$ and $L$ is uniformly elliptic in $D$, particularizing (3.12) at $h_{n}$, passing to the limit as $n \rightarrow \infty$ and using (3.9) gives

$$
\int_{D} V \Omega_{0}^{2}=V_{L} \int_{D} \Omega_{0}^{2}
$$

Therefore, $\Omega_{0}=0$ in $D_{+}$. This completes the proof.

Remark 3.3. (a) If $V \in C(D)$ and $\left|D \backslash D_{+}\right|=0$, then (3.8) implies

$$
\lim _{h \downarrow 0}\left\|\Omega_{h}\right\|_{L^{2}(D)}=0
$$

and hence, due to (3.7), no subsequence of $\Omega_{h}$ can converge in $W_{0}^{1,2}(D)$ to the $L^{2}$-limit of the ground states.

(b) When $V$ is continuous, a variant of Theorem 3.1 is to normalize $\Omega_{h}$ so that

$$
\left\|\Omega_{h}\right\|_{L^{\infty}(D)}=1 \text {. }
$$

Then, $\Omega_{h} \downarrow 0$ uniformly on any compact subset of $\bar{D} \cap D_{+}$. Indeed, if not, we rescale and use a blow-up argument near a point $x_{h}$ where $\Omega_{h}\left(x_{h}\right) \geq a>0$. If $x_{h} \rightarrow y_{0}$ as $h \rightarrow 0$, we obtain a solution of

$$
-\sum_{i, j=1}^{N} a_{i j}\left(y_{0}\right) \partial_{i} \partial_{j} w+V\left(y_{0}\right) w=V_{L} w
$$

on either $\mathbf{R}^{N}$ or a half-space, which is bounded and non-negative and satisfies $w\left(y_{0}\right) \geq a>0$. Moreover, we can use a change of variables so that $a_{i j}\left(y_{0}\right)=\delta_{i j}$ (cf. [7). In both cases $w=0$ is the unique bounded non-negative solution of this equation. This contradiction completes the proof. The result is well known for the full space case; it can be proved for instance by obtaining a linear differential equation for the average of $w$ on spheres. In the half space case one can show that $\partial_{j} w>0$ provided the half-space is $x_{j}>0$ (by a variant of a result in [6]), and then show that $\lim _{x_{j} \rightarrow \infty} w$ satisfies a full space equation in one lower dimension. 


\section{Decay RATe For potentials With SEVERAL DEgEnERATE WELls}

The main result of this section is the following.

Theorem 4.1. Suppose $a_{i j} \in W^{1, \infty}(D), b_{i}, c \in L^{\infty}(D), V \in L^{\infty}\left(\mathbf{R}^{N}\right)$,

$$
\left\{x \in D: V(x)=V_{L}\right\}=\left\{x_{0}, \ldots, x_{p}\right\}, \quad p \geq 0,
$$

and for each $i=0, \ldots, p$,

$V(x)=V_{L}+\left\|x-x_{i}\right\|^{\alpha_{i}} g_{i}(\omega)+o\left(\left\|x-x_{i}\right\|^{\alpha_{i}}\right) \quad$ as $x \rightarrow x_{i}, \quad \omega=\frac{x-x_{i}}{\left\|x-x_{i}\right\|}$,

for some $\alpha_{i}>0$ such that

$$
\alpha:=\alpha_{0}>\max _{1 \leq i \leq p} \alpha_{i}
$$

where each $g_{i}: S^{N-1} \rightarrow \mathbf{R}_{+}$is bounded and positive (bounded away from zero). Here $S^{N-1}$ stands for the $(N-1)$-dimensional unit sphere. Also, suppose

$$
\liminf _{\|x\| \rightarrow \infty} V(x)>V_{L} \text {. }
$$

Then

$$
\lim _{h \downarrow 0} \frac{\sigma_{1}^{D}\left[h^{2} L+V\right]-V_{L}}{h^{\frac{2 \alpha}{\alpha+2}}}=\sigma_{1}^{\mathbf{R}^{N}}\left[-\sum_{i, j=1}^{N} a_{i j}\left(x_{0}\right) \partial_{i} \partial_{j}+W\right]>0,
$$

where $W$ is the potential defined by

$$
W(y)=\|y\|^{\alpha} g_{0}(\omega), \quad \omega=\frac{y}{\|y\|}, \quad y \in \mathbf{R}^{N} .
$$

Moreover, if $\Omega_{h}$ is the principal eigenfunction associated with $\sigma_{1}^{D}\left[h^{2} L+V\right]$ normalized so that

$$
\left\|\Omega_{h}\right\|_{L^{2}(D)}=1
$$

then

$$
\lim _{h \downarrow 0}\left\|h^{\frac{N}{\alpha+2}} \Omega_{h}\left(x_{0}+h^{\frac{2}{\alpha+2}} \cdot\right)-\Psi_{0}(\cdot)\right\|_{L^{2}\left(\mathbf{R}^{N}\right)}=0,
$$

where $\Psi_{0}$ stands for the principal eigenfunction associated with

$$
\sigma_{1}^{\mathbf{R}^{N}}\left[-\sum_{i, j=1}^{N} a_{i j}\left(x_{0}\right) \partial_{i} \partial_{j}+W\right]
$$

normalized so that

$$
\int_{\mathbf{R}^{N}} \Psi_{0}^{2}(y) d y=1
$$

Proof. Thanks to Theorem 3.1 without loss of generality we can assume that

$$
V_{L}=0 \text {. }
$$

By the definition of $\Omega_{h}$ we have

$$
-h^{2} \sum_{i, j=1}^{N} a_{i j} \partial_{i} \partial_{j} \Omega_{h}+h^{2} \sum_{j=1}^{N} b_{j} \partial_{j} \Omega_{h}+h^{2} c \Omega_{h}+V \Omega_{h}=\sigma_{1}^{D}\left[h^{2} L+V\right] \Omega_{h}
$$


in $D$, and $\Omega_{h}=0$ on $\partial D$. Thus, making the change of variable

$$
y=h^{\frac{-2}{\alpha+2}}\left(x-x_{0}\right), \quad \Psi_{h}(y)=h^{\frac{N}{\alpha+2}} \Omega_{h}(x), \quad x \in D,
$$

we see that the new function $\Psi_{h}(y)$ satisfies

$$
\begin{aligned}
& -\sum_{i, j=1}^{N} a_{i j}\left(x_{0}+h^{\frac{2}{\alpha+2}} y\right) \partial_{i} \partial_{j} \Psi_{h}+h^{\frac{2}{\alpha+2}} \sum_{j=1}^{N} b_{j}\left(x_{0}+h^{\frac{2}{\alpha+2}} y\right) \partial_{j} \Psi_{h} \\
& \quad+h^{\frac{4}{\alpha+2}} c\left(x_{0}+h^{\frac{2}{\alpha+2}} y\right) \Psi_{h}+h^{\frac{-2 \alpha}{\alpha+2}} V\left(x_{0}+h^{\frac{2}{\alpha+2}} y\right) \Psi_{h}=\frac{\sigma_{1}^{D}\left[h^{2} L+V\right]}{h^{\frac{2 \alpha}{\alpha+2}}} \Psi_{h}
\end{aligned}
$$

in

$$
D_{h}:=\left\{h^{\frac{-2}{\alpha+2}}\left(x-x_{0}\right): x \in D\right\},
$$

and $\Psi_{h}=0$ on $\partial D_{h}$. Moreover, from (4.6) and (4.9) the following holds:

$$
\int_{D_{h}} \Psi_{h}^{2}(y) d y=1
$$

Let $\mathcal{L}_{h}$ denote the differential operator defined by the left hand side of (4.10), i.e.

$$
\begin{aligned}
\mathcal{L}_{h}= & -\sum_{i, j=1}^{N} a_{i j}\left(x_{0}+h^{\frac{2}{\alpha+2}} y\right) \partial_{i} \partial_{j}+h^{\frac{2}{\alpha+2}} \sum_{j=1}^{N} b_{j}\left(x_{0}+h^{\frac{2}{\alpha+2}} y\right) \partial_{j} \\
& +h^{\frac{4}{\alpha+2}} c\left(x_{0}+h^{\frac{2}{\alpha+2}} y\right)+h^{\frac{-2 \alpha}{\alpha+2}} V\left(x_{0}+h^{\frac{2}{\alpha+2}} y\right),
\end{aligned}
$$

with domain

$$
\mathcal{D}\left(\mathcal{L}_{h}\right)=W_{0}^{1, p}\left(D_{h}\right) \cap W^{2, p}\left(D_{h}\right) .
$$

By the uniqueness of the principal eigenvalue, (4.10) implies

$$
\sigma_{1}^{D_{h}}\left[\mathcal{L}_{h}\right]=\frac{\sigma_{1}^{D}\left[h^{2} L+V\right]}{h^{\frac{2 \alpha}{\alpha+2}}} .
$$

Fix $R>0$ arbitrarily. Then, for $h$ sufficiently small, $B_{R}$ is a proper subdomain of $D_{h}$ and hence it follows from (4.13) that

$$
\frac{\sigma_{1}^{D}\left[h^{2} L+V\right]}{h^{\frac{2 \alpha}{\alpha+2}}}<\sigma_{1}^{B_{R}}\left[\mathcal{L}_{h}\right] .
$$

Moreover, it follows from (4.2) that

$$
h^{\frac{-2 \alpha}{\alpha+2}} V\left(x_{0}+h^{\frac{2}{\alpha+2}} y\right)=\|y\|^{\alpha} g_{0}(\omega)+o(1) \quad \text { as } h \downarrow 0
$$

uniformly in $y \in B_{R}$. Thus, by the same argument of the proof of Theorem 2.1 the operators $\mathcal{L}_{h}$ converge in $B_{R}$ as $h \downarrow 0$ to the operator $\mathcal{L}_{0}$ defined by

$$
\mathcal{L}_{0}:=-\sum_{i, j=1}^{N} a_{i j}\left(x_{0}\right) \partial_{i} \partial_{j}+W(y), \quad \mathcal{D}\left(\mathcal{L}_{0}\right):=W_{0}^{1, p}\left(B_{R}\right) \cap W^{2, p}\left(B_{R}\right),
$$

in the sense of [15], IV.2.4, for any $p \geq 2$. Hence,

$$
\lim _{h \downarrow 0} \sigma_{1}^{B_{R}}\left[\mathcal{L}_{h}\right]=\sigma_{1}^{B_{R}}\left[\mathcal{L}_{0}\right],
$$


and therefore, we find from (4.14) that

$$
\underset{h \downarrow 0}{\limsup } \frac{\sigma_{1}^{D}\left[h^{2} L+V\right]}{h^{\frac{2 \alpha}{\alpha+2}}} \leq \sigma_{1}^{B_{R}}\left[\mathcal{L}_{0}\right] .
$$

Set

$$
s\left(\mathcal{L}_{0}\right):=\inf \left\{\operatorname{Re} \lambda: \lambda \in \sigma\left(\mathcal{L}_{0}\right)\right\} .
$$

By formula (1.2) of [2], $s\left(\mathcal{L}_{0}\right)$ admits the following variational characterization:

$$
s\left(\mathcal{L}_{0}\right)=\inf \left\{\int_{\mathbf{R}^{N}}\left(\langle A \nabla \varphi, \nabla \varphi\rangle+W \varphi^{2}\right), \varphi \in \mathcal{C}_{0}^{\infty}\left(\mathbf{R}^{N}\right),\|\varphi\|_{L^{2}}=1\right\},
$$

where $A=\left(a_{i j}\left(x_{0}\right)\right)$ and $\langle\cdot, \cdot\rangle$ stands for the Euclidean product in $\mathbf{R}^{N}$. Therefore, since (4.17) is valid for any $R>0$, it follows from the variational characterization of $\sigma_{1}^{B_{R}}\left[\mathcal{L}_{0}\right]$ that

$$
\limsup _{h \downarrow 0} \frac{\sigma_{1}^{D}\left[h^{2} L+V\right]}{h^{\frac{2 \alpha}{\alpha+2}}} \leq s\left(\mathcal{L}_{0}\right) .
$$

In particular, the quotients

$$
\frac{\sigma_{1}^{D}\left[h^{2} L+V\right]}{h^{\frac{2 \alpha}{\alpha+2}}}
$$

are uniformly bounded above. Now, we show that these quotients are also uniformly bounded below. Multiplying (4.10) by $\Psi_{h}$, integrating in $D_{h}$, applying the formula of integration by parts and using (4.11) -(4.13) gives

$$
\begin{aligned}
& \sum_{i, j=1}^{N} \int_{D_{h}} a_{i j}\left(x_{0}+h^{\frac{2}{\alpha+2}} y\right) \partial_{i} \Psi_{h} \partial_{j} \Psi_{h}+h^{\frac{2}{\alpha+2}} \sum_{j=1}^{N} \int_{D_{h}} B_{j}\left(x_{0}+h^{\frac{2}{\alpha+2}} y\right) \Psi_{h} \partial_{j} \Psi_{h} \\
& +h^{\frac{4}{\alpha+2}} \int_{D_{h}} c\left(x_{0}+h^{\frac{2}{\alpha+2}} y\right) \Psi_{h}^{2}+h^{\frac{-2 \alpha}{\alpha+2}} \int_{D_{h}} V\left(x_{0}+h^{\frac{2}{\alpha+2}} y\right) \Psi_{h}^{2}=\sigma_{1}^{D_{h}}\left[\mathcal{L}_{h}\right],
\end{aligned}
$$

where the new coefficients $B_{j}$ are those given by (3.10). By (4.11) and the Hölder inequality we find that

$$
\left|\sum_{j=1}^{N} \int_{D_{h}} B_{j}\left(x_{0}+h^{\frac{2}{\alpha+2}} y\right) \Psi_{h} \partial_{j} \Psi_{h}\right| \leq \beta\left\|\nabla \Psi_{h}\right\|_{L^{2}\left(D_{h}\right)},
$$

where

$$
\beta:=\sup _{x \in D}\|B(x)\| .
$$

Thus, by (4.13), (4.19) and (4.21) it follows from (4.20) that for all $\epsilon>0$ there exists $h_{0}=h(\epsilon)>0$ such that for $h \in\left(0, h_{0}\right)$ we have

$$
\begin{aligned}
& \nu\left\|\nabla \Psi_{h}\right\|_{L^{2}\left(D_{h}\right)}^{2}-\beta h^{\frac{2}{\alpha+2}}\left\|\nabla \Psi_{h}\right\|_{L^{2}\left(D_{h}\right)} \\
& \quad+h^{\frac{-2 \alpha}{\alpha+2}} \int_{D_{h}} V\left(x_{0}+h^{\frac{2}{\alpha+2}} y\right) \Psi_{h}^{2} \leq s\left(\mathcal{L}_{0}\right)+\epsilon-c_{L} h^{\frac{4}{\alpha+2}},
\end{aligned}
$$

where $c_{L}:=\operatorname{ess} \inf c$ and $\nu>0$ is the ellipticity constant of $L$. Since the right hand side of this inequality is bounded above uniformly in $h \in\left(0, h_{0}\right)$, it is easily seen 
that there exists a constant $K>0$ such that

$$
\left\|\nabla \Psi_{h}\right\|_{L^{2}\left(D_{h}\right)} \leq K, \quad h^{\frac{-2 \alpha}{\alpha+2}} \int_{D_{h}} V\left(x_{0}+h^{\frac{2}{\alpha+2}} y\right) \Psi_{h}^{2} \leq K, \quad \forall h \in\left(0, h_{0}\right) .
$$

Moreover, going back to (4.20) and using (4.22), the same argument as above also shows that $\sigma_{1}^{D_{h}}\left[\mathcal{L}_{h}\right]$ are uniformly bounded below. We now show that

$$
\lim _{h \downarrow 0} \sigma_{1}^{D_{h}}\left[\mathcal{L}_{h}\right]=\lim _{h \downarrow 0} \frac{\sigma_{1}^{D}\left[h^{2} L+V\right]}{h^{\frac{2 \alpha}{\alpha+2}}}=s\left(\mathcal{L}_{0}\right) .
$$

To see (4.23) we argue by contradiction. By 4.19), if (4.23) is not true, then there exist $\lambda_{0} \in \mathbf{R}$ and a sequence $\left(h_{n}\right), n \geq 1$, such that $\lim _{n \rightarrow \infty} h_{n}=0$ and

$$
\lim _{n \rightarrow \infty} \sigma_{1}^{D_{h_{n}}}\left[\mathcal{L}_{h_{n}}\right]=\lambda_{0}<s\left(\mathcal{L}_{0}\right) .
$$

By (4.22), we can assume that $\left(h_{n}\right)$ has been chosen so that

$$
\lim _{n \rightarrow \infty} \Psi_{h_{n}}=\Psi_{0} \in W^{1,2}\left(\mathbf{R}^{N}\right) \text { weakly in } W^{1,2}\left(\mathbf{R}^{N}\right) \text { and strongly in } L^{2}\left(\mathbf{R}^{N}\right) .
$$

We claim that $\Psi_{0}$ is a weak solution of

$$
-\sum_{i, j=1}^{N} a_{i j}\left(x_{0}\right) \partial_{i} \partial_{j} \Psi_{0}+W \Psi_{0}=\lambda_{0} \Psi_{0}
$$

To show this, pick $\varphi \in C_{0}^{\infty}\left(\mathbf{R}^{N}\right)$. Multiplying the equation of $\Psi_{h_{n}}$ by $\varphi$, integrating on $D_{h_{n}}$ and applying the formula of integration by parts gives

$$
\begin{aligned}
& \sum_{i, j=1}^{N} \int_{D_{h_{n}}} a_{i j}\left(x_{0}+h_{n}^{\frac{2}{\alpha+2}} y\right) \partial_{i} \Psi_{h_{n}} \partial_{j} \varphi \\
& \quad+h_{n}^{\frac{2}{\alpha+2}} \sum_{j=1}^{N} \int_{D_{h_{n}}} B_{j}\left(x_{0}+h_{n}^{\frac{2}{\alpha+2}} y\right) \varphi \partial_{j} \Psi_{h_{n}} \\
& \quad+h_{n}^{\frac{4}{\alpha+2}} \int_{D_{h_{n}}} c\left(x_{0}+h_{n}^{\frac{2}{\alpha+2}} y\right) \varphi \Psi_{h_{n}}+h_{n}^{\frac{-2 \alpha}{\alpha+2}} \int_{D_{h_{n}}} V\left(x_{0}+h_{n}^{\frac{2}{\alpha+2}} y\right) \varphi \Psi_{h_{n}} \\
& =\sigma_{1}^{D_{h_{n}}}\left[\mathcal{L}_{h_{n}}\right] \int_{D_{h_{n}}} \varphi \Psi_{h_{n}} .
\end{aligned}
$$

Thus, passing to the limit as $n \rightarrow \infty$ in (4.27), it follows from (4.2), (4.24) and (4.25) that

$$
\sum_{i, j=1}^{N} \int_{\mathbf{R}^{N}} a_{i j}\left(x_{0}\right) \partial_{i} \Psi_{0} \partial_{j} \varphi+\int_{\mathbf{R}^{N}}\|y\|^{\alpha} g_{0}(\omega) \varphi \Psi_{0}=\lambda_{0} \int_{\mathbf{R}^{N}} \varphi \Psi_{0} .
$$

Therefore, 4.26) holds true. Moreover, since $\lambda_{0}<s\left(\mathcal{L}_{0}\right)$, 4.18) implies that

$$
\Psi_{0}=0 \text {. }
$$

On the other hand, given $\delta>0$ arbitrary, there exists $\epsilon>0$ such that

$$
h^{\frac{-2 \alpha}{\alpha+2}} V\left(x_{0}+h^{\frac{2}{\alpha+2}} y\right) \geq \delta \quad \forall y \in \hat{D}_{h}:=D_{h} \backslash \bigcup_{i=0}^{p} B_{\epsilon}\left(y_{i}\right)
$$


where

$$
y_{i}=h^{\frac{-2}{\alpha+2}}\left(x_{i}-x_{0}\right), \quad i=0, \ldots, p .
$$

Moreover, (4.22) implies

$$
h^{\frac{-2 \alpha}{\alpha+2}} \int_{\hat{D}_{h}} V\left(x_{0}+h^{\frac{2}{\alpha+2}} y\right) \Psi_{h}^{2}+\sum_{i=0}^{p} h^{\frac{-2 \alpha}{\alpha+2}} \int_{B_{\epsilon}\left(y_{i}\right)} V\left(x_{0}+h^{\frac{2}{\alpha+2}} y\right) \Psi_{h}^{2} \leq K,
$$

uniformly in $h \in\left(0, h_{0}\right)$. Hence,

$$
\int_{\hat{D}_{h}} \Psi_{h}^{2} \leq \frac{K}{\delta}
$$

and if we choose $\delta$ sufficiently large, it follows from (4.11) that

$$
\sum_{i=0}^{p} \int_{B_{\epsilon}\left(y_{i}\right)} \Psi_{h_{n}}^{2} \geq \frac{1}{2},
$$

for $n$ sufficiently large. Thus, for some $i \in\{0, \ldots, p\}$ we have

$$
\int_{B_{\epsilon}\left(y_{i}\right)} \Psi_{h_{n}}^{2} \geq \frac{1}{2 p}
$$

We claim that $i=0$. Indeed, since the sequence $\Psi_{h_{n}}\left(y-y_{i}\right)$ is bounded in $W^{1,2}\left(B_{\epsilon}(0)\right)$, without loss of generality we can assume that

$$
\lim _{n \rightarrow \infty} \Psi_{h_{n}}\left(y-y_{i}\right)=\hat{\Psi}_{0}
$$

strongly in $L^{2}\left(B_{\epsilon}(0)\right)$. Moreover, by (4.32), $\hat{\Psi}_{0} \neq 0$. If $i \neq 0$, then for $y \neq y_{i}$ we have

$$
\lim _{n \rightarrow \infty} h_{n}^{\frac{-2 \alpha}{\alpha+2}} V\left(x_{0}+h_{n}^{\frac{2}{\alpha+2}}\left(y-y_{i}\right)\right)=\infty,
$$

since $\left\|x-x_{i}\right\| \leq \epsilon h^{\frac{2}{\alpha+2}}$ and $\alpha>\alpha_{i}$. Moreover, if $0<\nu<\epsilon$, then

$$
h_{n}^{\frac{-2 \alpha}{\alpha+2}} \int_{B_{\epsilon}(0) \backslash B_{\nu}(0)} V\left(x_{0}+h_{n}^{\frac{2}{\alpha+2}}\left(y-y_{i}\right)\right) \Psi_{h_{n}}^{2}\left(y-y_{i}\right) \leq K,
$$

and so passing to the limit as $n \rightarrow \infty$ gives

$$
\int_{B_{\epsilon}(0) \backslash B_{\nu}(0)} \hat{\Psi}_{0}^{2}=0
$$

and therefore $\hat{\Psi}_{0}=0$ a.e. on $B_{\epsilon}(0)$, which is impossible. This contradiction shows that $i=0$. Now, (4.32) gives

$$
\int_{B_{\epsilon}(0)} \Psi_{h_{n}}^{2} \geq \frac{1}{2 p}
$$

and, passing to the limit as $n \rightarrow \infty$, we find from (4.25) that

$$
\int_{B_{\epsilon}(0)} \Psi_{0}^{2} \geq \frac{1}{2 p}
$$

This relation contradicts (4.28) and therefore shows (4.23). Observe that the previous argument also shows (4.7), since $s\left(\mathcal{L}_{0}\right)$ is a simple eigenvalue of $\mathcal{L}_{0}$ in $L^{2}\left(\mathbf{R}^{N}\right)$ 
and the argument works out along any sequence $h_{n} \downarrow 0$. We should point out that in the statement of the theorem we have used the notation

$$
\sigma_{1}^{\mathbf{R}^{N}}\left[\mathcal{L}_{0}\right]=s\left(\mathcal{L}_{0}\right) .
$$

It remains to show that $s\left(\mathcal{L}_{0}\right)>0$. This follows easily from 4.20). Indeed, passing to the limit as $n \rightarrow \infty$ and using Fatou's lemma, we find that

$$
\int_{\mathbf{R}^{N}}\left(\left\langle A \nabla \Psi_{0}, \Psi_{0}\right\rangle+\|y\|^{\alpha} \Psi_{0}^{2}\right) \leq s\left(\mathcal{L}_{0}\right),
$$

and so necessarily $s\left(\mathcal{L}_{0}\right)>0$. This can be also shown from the abstract theory in 2 and [4]. This completes the proof.

Remark 4.2. If $\partial D$ is of class $C^{1}$, then in Theorem 4.1 we can allow $x_{0} \in \partial D$. The only difference is that the limit problem is a half space problem, instead of a full space problem.

Corollary 4.3. Suppose $a_{i j} \in W^{1, \infty}(D), b_{i}, c \in L^{\infty}(D), V \in L^{\infty}\left(\mathbf{R}^{N}\right)$,

$$
\left\{x \in D: V(x)=V_{L}\right\}=\left\{x_{0}, \ldots, x_{p}\right\}, \quad p \geq 0 .
$$

and for each $i=0, \ldots, p$,

$V(x)=V_{L}+\left\|x-x_{i}\right\|^{\alpha_{i}} g_{i}(\omega)+o\left(\left\|x-x_{i}\right\|^{\alpha_{i}}\right) \quad$ as $x \rightarrow x_{i}, \quad \omega=\frac{x-x_{i}}{\left\|x-x_{i}\right\|}$,

where $\alpha_{i}>0$ and $g_{i}: S^{N-1} \rightarrow \mathbf{R}_{+}$is bounded and positive. Set

$$
\alpha:=\max _{0 \leq i \leq p} \alpha_{i}
$$

and let $\left\{i_{1}, \ldots, i_{q}\right\} \subset\{0, \ldots, p\}$ be the set of indices for which

$$
\alpha_{j}=\alpha, \quad j=i_{1}, \ldots, i_{q} .
$$

Then

$$
\lim _{h \downarrow 0} \frac{\sigma_{1}^{D}\left[h^{2} L+V\right]-V_{L}}{h^{\frac{2 \alpha}{\alpha+2}}}=\min _{1 \leq j \leq q} s\left(\mathcal{L}_{j}\right)
$$

where

$$
\mathcal{L}_{j}=-\sum_{k, \ell=1}^{N} a_{k \ell}\left(x_{i_{j}}\right) \partial_{k} \partial_{\ell}+\|y\|^{\alpha} g_{i_{j}}(\omega), \quad 1 \leq j \leq q .
$$

Thanks to Theorem 4.1, (4.33) is true if $q=1$. This corollary is an extension of Theorem 4.1 to cover the case when $q \geq 2$.

Proof. Pick $j \in\{1, \ldots, q\}$. Let $V_{j}$ denote any potential which is equal to $V$ within a sufficiently small neighborhood of $x_{i_{j}}$, is bounded away from $V_{L}$ outside that neighborhood, and $V_{j} \geq V$. By the monotonicity of the principal eigenvalue with respect to the potential we have

$$
\sigma_{1}^{D}\left[h^{2} L+V\right] \leq \sigma_{1}^{D}\left[h^{2} L+V_{j}\right]
$$

Moreover, thanks to Theorem 4.1

$$
\lim _{h \downarrow 0} \frac{\sigma_{1}^{D}\left[h^{2} L+V_{j}\right]-V_{L}}{h^{\frac{2 \alpha}{\alpha+2}}}=s\left(\mathcal{L}_{j}\right) .
$$


Thus,

$$
\limsup _{h \downarrow 0} \frac{\sigma_{1}^{D}\left[h^{2} L+V\right]-V_{L}}{h^{\frac{2 \alpha}{\alpha+2}}} \leq s\left(\mathcal{L}_{j}\right) .
$$

Since this is true for any $j$, the following holds:

$$
\limsup _{h \downarrow 0} \frac{\sigma_{1}^{D}\left[h^{2} L+V\right]-V_{L}}{h^{\frac{2 \alpha}{\alpha+2}}} \leq \min _{1 \leq j \leq q} s\left(\mathcal{L}_{j}\right) .
$$

To complete the proof, pick $j_{0} \in\{1, \ldots, q\}$ such that

$$
s\left(\mathcal{L}_{j_{0}}\right):=\min _{1 \leq j \leq q} s\left(\mathcal{L}_{j}\right) .
$$

Then, the blowing-up scaling

$$
y=h^{\frac{-2}{\alpha+2}}\left(x-x_{i_{0}}\right), \quad \Psi_{h}(y)=h^{\frac{N}{\alpha+2}} \Omega_{h}(x), \quad x \in D,
$$

works out to get the first part of the proof of Theorem 4.1 Moreover, thanks to (4.34), if (4.33) fails to be true, then there exist $\lambda_{0} \in \mathbf{R}$ and a sequence $\left(h_{n}\right), n \geq 1$, such that $\lim _{n \rightarrow \infty} h_{n}=0$ and

$$
\lim _{n \rightarrow \infty} \frac{\sigma_{1}^{D}\left[h_{n}^{2} L+V\right]-V_{L}}{h_{n}^{\frac{2 \alpha}{\alpha+2}}}=\lambda_{0}<s\left(\mathcal{L}_{j_{0}}\right) .
$$

Using the same argument as in the proof of Theorem 4.1, 4.35) leads to a contradiction. This completes the proof.

Also, the proof of Theorem 4.1 can be adapted to cover some special cases where $V_{L}$ is taken along infinitely many points. Indeed, assume that

$$
\left\{x \in D: V(x)=V_{L}\right\}=\left\{x_{n}, n \geq 1\right\},
$$

where $x_{n}$ is a sequence such that

$$
\lim _{n \rightarrow \infty} x_{n}=x_{0} \in \bar{D} \text {. }
$$

Suppose in addition that for each $n \geq 1$

$$
V(x)=V_{L}+\left\|x-x_{n}\right\|^{n} g_{n}(\omega)+o\left(\left\|x-x_{n}\right\|^{n}\right) \quad \text { as } x \rightarrow x_{n}, \quad \omega=\frac{x-x_{n}}{\left\|x-x_{n}\right\|},
$$

where $g_{n}: S^{N-1} \rightarrow \mathbf{R}_{+}$is bounded and positive. Then, using the same argument as in the proof of Theorem 4.1, we find that for each $n \geq 1$

$$
\limsup _{h \downarrow 0} \frac{\sigma_{1}^{D}\left[h^{2} L+V\right]-V_{L}}{h^{\frac{2 n}{n+2}}} \leq s\left(-\sum_{i, j=1}^{N} a_{i j}\left(x_{n}\right) \partial_{i} \partial_{j}+\|y\|^{n} g_{n}(\omega)\right) .
$$

Since $\lim _{n \rightarrow \infty} \frac{2 n}{n+2}=2$, (4.36) suggests that $h^{2}$ is the exact decay order of the principal eigenvalue to $V_{L}$. But this is not the case, as the following version of Theorem 6.4 in [16] shows.

Theorem 4.4. Suppose $a_{i j} \in W^{1, \infty}(D), b_{i}, c \in L^{\infty}(D), V \in L^{\infty}\left(\mathbf{R}^{N}\right)$ and

$$
\left|\left\{x \in D: V(x)=V_{L}\right\}\right|=0 .
$$

Then,

$$
\lim _{h \downarrow 0} \frac{\sigma_{1}^{D}\left[h^{2} L+V\right]-V_{L}}{h^{2}}=\infty .
$$


The details of the proof can be adapted mutatis mutandis from the proof of Theorem 6.4 in [16], and so we will omit them. Due to Theorem 4.4 it would be of great interest to ascertain the exact decay rate in the previous example.

\section{Decay Rate For the Case When $V$ Vanishes on a SUbdomain}

The main result of this section is the following.

Theorem 5.1. Suppose $a_{i j} \in W^{1, \infty}(D), b_{i}, c \in L^{\infty}(D)$ and $V \in L^{\infty}(D)$. Set

$$
D_{+}:=\left\{x \in D: V(x)>V_{L}\right\}
$$

and suppose that

$$
D_{0}:=D \backslash \bar{D}_{+}
$$

is an open subset of $D$ which is stable in the sense of Babuška and Výborný [3]. Then,

$$
\lim _{h \downarrow 0} \frac{\sigma_{1}^{D}\left[h^{2} L+V\right]-V_{L}}{h^{2}}=\sigma_{1}^{D_{0}}[L] .
$$

Moreover, if $\Omega_{h}>0$ stands for the principal eigenfunction associated with $\sigma_{1}^{D}\left[h^{2} L+V\right]$, normalized by

$$
\int_{D} \Omega_{h}^{2}=1
$$

then, for any sequence $h_{n} \rightarrow 0$ as $n \rightarrow \infty$, there exists a subsequence of $\Omega_{h_{n}}$, relabeled by $n$, such that

$$
\lim _{n \rightarrow \infty}\left\|\Omega_{h_{n}}-\hat{\Omega}_{0}\right\|_{L^{2}(D)}=0
$$

where $\hat{\Omega}_{0}$ is the extension by zero of a principal eigenfunction $\Omega_{0}$ associated with $\sigma_{1}^{D_{0}}[L]$ normalized by

$$
\int_{D_{0}} \Omega_{0}^{2}=1
$$

Relation (5.1) was found in Theorems 6.2, 6.7 of [16] for a less general class of operators and subdomains. The behavior of the ground states is new. Our proof of (5.3) will provide us simultaneously with a striking proof of (5.1). We should point out that since $D_{0}$ is not assumed to be connected, the principal eigenvalue is not necessarily simple. This is why $\hat{\Omega}_{0}$ is the extension by zero of some of its associated eigenfunctions. If $D_{0}$ is connected, then $\hat{\Omega}_{0}$ is uniquely determined and

$$
\lim _{h \downarrow 0}\left\|\Omega_{h}-\hat{\Omega}_{0}\right\|_{L^{2}(D)}=0 .
$$

Proof of Theorem 5.1. Thanks to Theorem 3.1, we can assume

$$
V_{L}=0 \text {. }
$$

The function $\Omega_{h}$ satisfies

$$
-\sum_{i, j=1}^{N} a_{i j} \partial_{i} \partial_{j} \Omega_{h}+\sum_{j=1}^{N} b_{j} \partial_{j} \Omega_{h}+c \Omega_{h}^{2}+\frac{V}{h^{2}} \Omega_{h}=\sigma_{1}^{D}\left[L+\frac{V}{h^{2}}\right] \Omega_{h}
$$


in $D$, and $\Omega_{h}=0$ on $\partial D$. Multiplying (5.4) by $\Omega_{h}$, integrating on $D$, applying the formula of integration by parts and using (5.2) gives

$$
\sum_{i, j=1}^{N} \int_{D} a_{i j} \partial_{i} \Omega_{h} \partial_{j} \Omega_{h}+\sum_{j=1}^{N} \int_{D} B_{j} \Omega_{h} \partial_{j} \Omega_{h}+\int_{D} c \Omega_{h}+\int_{D} \frac{V}{h^{2}} \Omega_{h}^{2}=\sigma_{1}^{D}\left[L+\frac{V}{h^{2}}\right],
$$

where the new coefficients $B_{j}$ are those given by (3.10). Since $D_{0}$ is a proper subdomain of $D$ and $V=0$ on $D_{0}$, we have

$$
\sigma_{1}^{D}\left[L+\frac{V}{h^{2}}\right]<\sigma_{1}^{D_{0}}[L] .
$$

In particular,

$$
\limsup _{h \downarrow 0} \sigma_{1}^{D}\left[L+\frac{V}{h^{2}}\right] \leq \sigma_{1}^{D_{0}}[L] .
$$

Moreover, by the Hölder's inequality

$$
\left|\sum_{j=1}^{N} \int_{D} B_{j} \Omega_{h} \partial_{j} \Omega_{h}\right| \leq \beta\left\|\nabla \Omega_{h}\right\|_{L^{2}(D)},
$$

where

$$
\beta:=\sup _{x \in D}\|B(x)\|, \quad B=\left(B_{i}\right) .
$$

Thus substituting (5.6) and (5.8) into (5.5) gives

$$
\nu\left\|\nabla \Omega_{h}\right\|_{L^{2}(D)}^{2}-\beta\left\|\nabla \Omega_{h}\right\|_{L^{2}(D)}+\int_{D} \frac{V}{h^{2}} \Omega_{h}^{2} \leq \sigma_{1}^{D_{0}}[L]-c_{L},
$$

where $c_{L}:=\operatorname{ess} \inf c$ and $\nu>0$ is the ellipticity constant of $L$. From this inequality it is easily seen that there exists a constant $K>0$ such that

$$
\left\|\nabla \Omega_{h}\right\|_{L^{2}(D)} \leq K, \quad \int_{D} \frac{V}{h^{2}} \Omega_{h}^{2} \leq K, \quad \forall h>0 .
$$

Moreover, going back to (5.5) and using (5.9), the same argument as above also shows that there exists $M \in \mathbf{R}$ such that

$$
\sigma_{1}^{D}\left[L+\frac{V}{h^{2}}\right] \geq M \quad \forall h>0 .
$$

We now show (5.1). The argument will follow by contradiction. If (5.1) is not true, by (5.7) and (5.10) there exist $\lambda_{0} \in \mathbf{R}$ and a sequence $\left(h_{n}\right), n \geq 1$, such that $\lim _{n \rightarrow \infty} h_{n}=0$ and

$$
\lim _{n \rightarrow \infty} \sigma_{1}^{D}\left[L+\frac{V}{h_{n}^{2}}\right]=\lambda_{0}<\sigma_{1}^{D_{0}}[L] .
$$

Moreover, thanks to (5.9) the sequence $\left(h_{n}\right)$ can be chosen so that

$$
\lim _{n \rightarrow \infty} \Omega_{h_{n}}=\Psi_{0} \in W^{1,2}(D) \quad \text { weakly in } W^{1,2}(D),
$$

and strongly in $L^{2}(D)$, for some $\Psi_{0}$. It is clear that $\Psi_{0} \geq 0$ and that

$$
\int_{D} \Psi_{0}^{2}=1
$$

By Theorem 3.2, $\Psi_{0}=0$ in $D_{+}$. Thus, since $D_{0}$ is stable,

$$
\Psi_{0} \in W_{0}^{1,2}\left(D_{0}\right) \text {. }
$$


To prove that $\Psi_{0}$ is a distributional solution, pick $\varphi \in C_{0}^{\infty}\left(D_{0}\right)$. Multiplying the equation of $\Omega_{h_{n}}$ by $\varphi$, integrating on $D$ and applying the formula of integration by parts gives

$$
\sum_{i, j=1}^{N} \int_{D_{0}} a_{i j} \partial_{i} \Omega_{h_{n}} \partial_{j} \varphi+\sum_{j=1}^{N} \int_{D_{0}} B_{j} \varphi \partial_{j} \Omega_{h_{n}}+\int_{D_{0}} c \varphi \Omega_{h_{n}}=\sigma_{1}^{D}\left[L+\frac{V}{h_{n}^{2}}\right] \int_{D_{0}} \varphi \Omega_{h_{n}}
$$

Thus, passing to the limit as $n \rightarrow \infty$, we find from (5.11) that

$$
\sum_{i, j=1}^{N} \int_{D_{0}} a_{i j} \partial_{i} \Psi_{0} \partial_{j} \varphi+\sum_{j=1}^{N} \int_{D_{0}} B_{j} \varphi \partial_{j} \Psi_{0}+\int_{D_{0}} c \varphi \Psi_{0}=\lambda_{0} \int_{D_{0}} \varphi \Psi_{0}
$$

Therefore, $\Psi_{0} \in W_{0}^{1,2}\left(D_{0}\right)$ is a weak solution of

$$
L \Psi_{0}=\lambda_{0} \Psi_{0}
$$

in $D_{0}$ subject to homogeneous Dirichlet boundary conditions. Thanks to Theorem 2.1 this is impossible, since we are assuming that $\lambda_{0}<\sigma_{1}^{D_{0}}[L]$. Therefore, (5.1) is true, and the same argument as before completes the proof of the remaining assertions of the theorem.

\section{ACKNOWLEDGEMENTS}

The authors thank to the Australian Research Council and the Spanish DGICYT PB93-0465 and DGES PB96-0621 for research support.

\section{REFERENCES}

[1] H. Amann and J. López-Gómez, A priori bounds and multiple solutions for superlinear indefinite elliptic problems, J. Diff. Eqns. 146 (1998), 336-374. [MR 99e:35057

[2] W. Arendt and C. J. K. Batty, Exponential stability of a diffusion equation with absorption, Diff. Int. Equns. 6 (1993), 1009-1024. MR 94k:35038

[3] I. Babuška and R. Výborný, Continuous dependence of eigenvalues on the domain, Czech. Math. J. 15 (1965), 169-178. MR 32:281

[4] C. J. K. Batty, Asymptotic stability of Schrödinger semigroups: Path integral methods, Math. Ann. 292 (1992), 457-492. MR 93g:47050

[5] E. N. Dancer, Some remarks on classical problems and fine properties of Sobolev spaces, Diff. Int. Eqns. 9 (1996), 437-446. MR 97e:35057

[6] E. N. Dancer, Some notes on the method of moving planes, Bull. Austral. Math. Soc. 46 (1992), 425-434. MR 93m:35080

[7] B. Gidas and J. Sprück, A priori bounds for positive solutions of nonlinear elliptic equations, Comm. Partial Diff. Equns. 6 (1981), 883-901. MR 82h:35033

[8] B. Helffer, Semi-classical Analysis for the Schrödinger Operator and Applications, Lectures Notes in Mathematics 1336, Springer 1988. MR 90c:81043

[9] B. Helffer and J. Sjöstrand, Multiple wells in the semiclassical limit, I, Comm. PDEs 9 (1984), 337-408. MR 86c:35113

[10] B. Helffer and J. Sjöstrand, Puits multiples en limite semi-classique, II. Interaction moléculaire, symétries, perturbation, Ann. Inst. H. Poincaré 42 (1985), 127-212. MR 87a:35142

[11] B. Helffer and J. Sjöstrand, Multiple wells in the semi-classical limit, III. Interaction through non-resonant wells, Math. Nach. 124 (1985), 263-313. MR 87i:35161

[12] P. Hess, Periodic-Parabolic Boundary Value Problems and Positivity, Pitman Res. Notes in Math., vol. 247, Longman, Harlow 1991. MR 92h:35001

[13] P. Hess and T. Kato, On some linear and nonlinear eigenvalue problems with an indefinite weight function, Comm. Part. Diff. Eqns. 5 (1980), 999-1030. [MR 81m:35102]

[14] P. D. Hislop and I. M. Sigal, Introduction to Spectral Theory with Applications to Schrödinger Operators, Appl. Math. Sc. 113, Springer, New York 1996. MR 98h:47003 
[15] T. Kato, Perturbation Theory for Linear Operators, Classics in Mathematics, Springer, Berlin 1995. MR 96a:47025

[16] J. López-Gómez, The maximum principle and the existence of principal eigenvalues for some linear weighted boundary value problems, J. Diff. Eqns. 127 (1996), 263-294. MR 97b:35037

[17] J. López-Gómez, On the linear damped wave equation, J. Diff. Eqns. 134 (1997), 26-45. MR 97m:35161

[18] J. López-Gómez, On the structure of the permanence region for competing species models with general diffusivities and transport effects, Disc. Cont. Dyn. Sys. 2 (1996), 525-542. MR 98d:92016

[19] A. Martínez and M. Rouleux, Effet tunnel entre puits dégénérés, Comm. Part. Diff. Eqns. 13 (1988), 1157-1187. MR 89h:35085

[20] B. de Pagter, Irreducible compact operators, Math. Z. 192 (1986), 149-153. MR 87d:47052

[21] H. H. Schaefer, Banach Lattices and Positive Operators, Springer, Berlin 1974. MR 54:11023

[22] B. Simon, Semiclassical analysis of low lying eigenvalues, I. Non-degenerate minima: Asymptotic expansions, Ann. Inst. Henri Poincaré A XXXVIII (1983), 12-37. MR 85m:81040

[23] B. Simon, Semiclassical analysis of low lying eigenvalues, II. Tunneling, Annals of Mathematics 120 (1984), 89-118. MR 87h:81045a

[24] B. Simon, Semiclassical analysis of low lying eigenvalues, III. Width of the ground state band in strongly coupled solids, Annals of Physics 158 (1984), 415-420. MR 87h:81045b

[25] B. Simon, Semiclassical analysis of low lying eigenvalues, IV. The flea of the elephant, J. Funct. Anal. 63 (1985), 123-136. MR 87h:81045c

[26] E. M. Stein, Singular Integrals and Differentiability Properties of Functions, Princeton Univ. Press, Princeton, N.J. 1970. MR 44:7280

Department of Mathematics, The University of Sydney, Sydney, N.S.W. 2006, AusTRALIA

E-mail address: normd@maths.usyd.edu.au

Departamento de Matemática Aplicada, Universidad Complutense de Madrid, 28040MADRID, SPAIN

E-mail address: julian@sunma4.mat.ucm.es 